Revista Sinapsis. Vol 1, Nro 16, junio de 2020 | ISSN 1390 - 9770

\title{
Práctica de autocuidado en paciente con diabetes mellitus tipo 2
}

\section{Self-care practice in patients with type 2 diabetes mellitus}

\section{Práctica de autocuidado en diabetes tipo 2}

Franklin Vite Solórzano, (1)

Jaime Humberto Flores García, (2)

Angy Vanessa Salazar Morillo, (3)

Jefferson Alexander Sornoza Párraga, ${ }^{(4)}$

(1) Universidad Técnica de Manabí. Ecuador. email: antuanvit@hotmail.com

(2) Universidad Técnica de Manabí. Ecuador. email: jaimeflores75@hotmail.com

(3) Universidad Técnica de Manabí. Ecuador. email: vanessa_angy.97@outloock.com

(4) Universidad Técnica de Manabí. Ecuador. email: jasp.13@hotmail.com

Contacto: antuanvit@hotmail.com

Recibido: 18-02-2020 Aprobado: 01-05-2020

\section{Resumen}

La investigación aborda la práctica de autocuidado en pacientes con diabetes mellitus tipo 2, la diabetes mellitus (DM) como un problema de salud con gran impacto que preocupa a profesionales sanitarios y a la sociedad en su conjunto. Se estima que una proporción de diabéticos no conoce su enfermedad y no ha concienciado la necesidad del autocuidado, además de no disponer de herramientas necesarias para ello. El objetivo es determinar las actividades de autocuidado practicadas por los pacientes con diabetes mellitus tipo 2 atendidos en los subcentros de la zona rural de Manabí-Ecuador. El estudio será descriptivo, transversal y prospectivo. La población serán los pacientes mayores de 18 años de edad registrados en las consultas de esas unidades de salud. La muestra se obtendrá en cada uno de los estratos, luego de aplicar la fórmula de Sierra Bravo. Se aplicarán instrumentos de recolección de datos validados a los pacientes con diabetes. Se concluye la detección de hábitos y conductas en beneficio a los pacientes en investigación.

Palabras clave: Autocuidado, pacientes diabéticos, hábitos, conductas

\footnotetext{
Abstract

The research addresses the practice of self-care in patients with type 2 diabetes mellitus, diabetes mellitus (DM) as a health problem with great impact
}

that concerns health professionals and society as a whole. It is estimated that a proportion of diabetics do not know their disease and are not aware of the need for self-care, in addition to not having the necessary tools to do so. The objective is to determine the self-care activities practiced by patients with type 2 diabetes mellitus treated in the sub-centers of the rural area of Manabi-Ecuador. The study will be descriptive, transversal and prospective. The population will be patients over 18 years of age registered in the consultations of these health units. The sample is requested in each of the strata, then the Sierra Bravo formula is applied. Validated data collection instruments will be applied to patients with diabetes. The detection of habits and behaviors for the benefit of research patients is concluded.

Keywords: Self-care, diabetic patients, habits, behaviors

\section{Introducción}

La Asociación Latinoamericana de Diabetes estima que la diabetes mellitus tipo 2 (DMT2), es una de las enfermedades más prevalentes en los adultos del continente y ocasiona costos muy elevados a los individuos, las familias y las naciones. Según un informe de esta asociación existen alrededor de 15 millones de personas con diabetes mellitus en Latinoamérica y esta cifra llegará a 20 millones en 10 años, mucho más de lo esperado por el simple 
incremento poblacional (Huañap, 2012).

La diabetes mellitus (DM) es un problema de salud con gran impacto que preocupa a profesionales sanitarios y a la sociedad en su conjunto. Contar con un paciente con DMT2 más activo y responsable con su salud, es considerado como una de las soluciones para combatir dicho problema (Naranjo, 2016).

La DMT2 es hoy en día, uno de los trastornos más frecuentes dentro de la patología general, afectando la calidad de vida de la persona que la padece y de la familia, eleva los costos de asistencia sanitaria en todos sus niveles, disminuye la esperanza de vida al nacer, produce complicaciones agudas, crónicas, discapacidad y muerte prematura. Su etiología tiene una base hereditaria, pero son los patrones de alimentación, el sedentarismo y otros estilos de vida medio ambientales, los que también contribuyen a que se desencadene, por lo que es indispensable que los pacientes tengan plena información sobre la enfermedad. La DMT2 aparece con mayor frecuencia a partir de los 40 años (por eso llamada diabetes del adulto). Para prevenir la aparición de la DMT2 en la población general y sobre todo en grupos vulnerables, es necesario que se conozcan medidas de autocuidado orientadas a la prevención; en caso de los pacientes detectados con esta enfermedad se debe tender a disminuir, prolongar o evitar la aparición de las complicaciones agudas y crónicas (Hernández, \& Aponte 2014).

Los conocimientos, prácticas y actitudes son elementos a considerar para mantener la salud, a si también mediante la concienciación del paciente, familia y comunidad sobre los cuidados en el control de su enfermedad. La historia natural de la DM se acompaña de una serie de manifestaciones a corto, mediano y largo plazo, las cuales, en cuanto a su desarrollo, frecuencia y severidad, están indiscutiblemente ligadas a la calidad del control metabólico y exposición a factores especialmente en los grupos más vulnerables, que tienden a sufrir complicaciones desmejorando la expectativa y calidad de vida (FPS, 2017).

$\mathrm{Al}$ considerar que las personas con DM presentan una condición crónica de salud, que exige cuidados permanentes para manutención de su calidad de vida y control metabólico, se percibe la necesidad de desarrollar habilidades de autocuidado para el manejo de la enfermedad. En particular para las personas con DMT2, cuya prevalencia está asociada al estilo de vida; razón por la cual, introducir cambios en los hábitos diarios puede constituir una estrategia efectiva en la prevención de la enfermedad y retardar las complicaciones crónicas provenientes de la misma (OMS, 2016)

El autocuidado, en la actualidad, constituye un tema de trascendencia, porque mediante su conocimiento y aplicación permiten incrementar la proactividad de los trabajadores en el campo de la salud. La práctica del autocuidado requiere, en gran medida, cambio de actitud frente a los nuevos retos de la vida moderna. Las nuevas concepciones de salud y promoción de la salud proponen una nueva forma de asumir el autocuidado con rostro humano, como estrategia fundamental para promover la vida y el bienestar; por eso se requiere el desarrollo de habilidades personales para optar decisiones saludables, lo cual constituye uno de los cinco componentes de la promoción de la salud (Velásquez, 2014).

La presencia del profesional de enfermería es particularmente importante cuando ocurren situaciones en que la persona tiene dificultad para responsabilizarse por el cuidado de sí misma. Así, para una actuación efectiva el profesional necesita conocer las variables relacionadas al autocuidado. Algunos estudios han abordado esos aspectos, investigando las variables relacionadas al acceso a la información y conocimiento, satisfacción con los servicios de salud y apoyo familiar, entre otros factores psicosociales relevantes Además, los pacientes pueden asumir un rol activo que se caracterice por seguir las pautas educativas sugeridas por su médico tratante y por las enfermeras o hacer caso omiso a estas, en cuyo caso su actitud contribuiría al deterioro de su estado de salud. En el caso de la DM, las complicaciones en los distintos órganos puede incluso llevar a discapacidad o a la muerte del paciente. En este caso, el personal de salud debe estar preparado y presto para orientar al paciente diabético en su responsabilidad de autocuidado para evitar las complicaciones. Ello, sin descuidar sus responsabilidades clínicas en el examen exhaustivo del estado físico de los mismos, lo que permitiría detectar precozmente si se presenta alguna de las complicaciones mencionadas y así limitar tanto los daños como las consecuencias negativas que podría significar el avance del deterioro de las condiciones en la supervivencia y la calidad de vida de los pacientes

La enfermería desde sus inicios se ha considerado como un producto social vinculado al arte de cuidar, por lo que responde a la necesidad de ayudar a las personas, cuando estas no tienen capacidad suficiente para proporcionarse a sí misma o a las 
personas que dependen de ellas, la calidad y cantidad de cuidados para mantener la vida, identificando los problemas de salud y las necesidades reales y/o potenciales de la persona, familia y comunidad que demandan cuidados, generando autonomía o dependencia como consecuencia de las actividades que asuma el enfermero

La enfermería como profesión en Ecuador es reglamentada por la Ley, la cual explicita que el profesional de enfermería tiene como fin "dar cuidado integral de salud a la persona, a la familia, la comunidad y a su entorno, para mantener prácticas de vida saludables que permitan salvaguardar un estado óptimo de salud en todas las etapas de la vida". En este marco normativo y en el propio de la autonomía universitaria, funcionan los programas de enfermería en Ecuador; los profesionales de la salud se forman para cuidar y proteger la salud del otro, lo cual se sustenta en los principios de la Ley de Ética en Enfermería. (Ley Orgánica de Salud. Código Ética, 2006)

Es de conocimiento general la relevancia del autocuidado a nivel internacional como pilar fundamental de la Atención Primaria en Salud con la promulgación de "Salud para todos en el año 2000", convirtiendo al autocuidado en un recurso sanitario fundamental de cualquier sistema de atención en salud. Aunque existe poca evidencia de la enseñanza del autocuidado, se resalta los beneficios de la estrategia didáctica en el ejercicio de la profesión al trabajar capacidades tales como: el autoaprendizaje, trabajo en equipo, pensamiento crítico, comunicación, actitudes y visión holística (Ministerio de Salud Pública. Código de Ética. Ecuador, 2013)

Día a día hombres y mujeres, niños, jóvenes, adultos $\mathrm{y}$ ancianos, inician sus jornadas con ilusiones, desafíos, entusiasmo y esperanza, en busca de un mejor futuro con bienestar y desarrollo. Considerar la salud como un derecho individual, en marcado en la constitución, implica generar condiciones en las cuales todos puedan vivir lo más saludablemente posible. Esto significa disponibilidad de servicios de salud, condiciones de trabajo saludables, vivienda adecuada y alimentos nutritivos, no solamente para la población urbana sino también para la rural. $\mathrm{La}$ población rural de Ecuador, merece mayor atención en su salud, esto significa desarrollar mejores acciones en promoción de la misma, prevención de las enfermedades, recuperación de la salud y rehabilitación, lo que requiere de un número suficiente de establecimientos, talento humano, medicamentos y suministros en los servicios públicos de salud dirigidos a las personas, familias y a las comunidades.

De acuerdo a lo expuesto, es importante determinar la relación entre las pautas educativas suministradas por el personal de salud y las prácticas de autocuidado en pacientes diabéticos del primer nivel de atención. Este estudio enfoca las variables relacionadas a la capacidad de autocuidado en personas atendidas en un sub servicio de salud en un contexto rural, donde existen limitantes de muchos tipos, a fin de ofrecer alternativas válidas para la restructuración de la atención de la población con DM en el norte de Ecuador, con el cual conocer las variables relacionadas al autocuidado posibilitará identificar las potenciales barreras para la adhesión de las personas con DMT2 al tratamiento instituido, así como proponer estrategias para el fortalecimiento del autocuidado en la población estudiada.

Es de gran importancia realizar un estudio sobre las practica de autocuidado sobre la DMT2 por motivo que es una enfermedad que con el pasar el tiempo atenta en gran mayoría a las personas siendo las más vulnerables las que superan los 40 años de edad, este estudio beneficiara a la sociedad en general y será de gran ayuda para los organismos pertinente que controlar la salud pública (MSP) ya que tendrán valores exactos del estilo de vida que llevan las personas que son atendidas en diversos sub centro de la provincia de Manabí.

El trabajo investigativo trata sobre la práctica de autocuidado en paciente con diabetes mellitus tipo 2 , el cual presenta como objetivo principal determinar las actividades de autocuidado practicadas por los pacientes con diabetes mellitus tipo 2, incluyendo la preparación del personal de salud en el cuidado de la misma en los subcentros de zonas rurales de ManabíEcuador.

\section{Materiales y métodos}

La presente investigación es un estudio, descriptivo transversal no experimental donde los hechos, serán descritos tal cual como son observados y al mismo tiempo estudiando la asociación entre variables.

Se procedió a la aplicación de los instrumentos de recolección de datos, previamente validados. Para el procesamiento de los datos recopilados, se utilizó el programa de Microsoft Excel

Para la recolección de datos se empleó el 
cuestionario sobre Capacidades de Autocuidado validado por Rodríguez y col. Para determinar la frecuencia de la diabetes mellitus tipo 2 en los Sub centros de salud de la zona norte de Manabí: Sub centro Canuto, San Antonio, Santa Martha, Santa Rita, Flavio Alfaro, Ricaurte, Cucuy, Eloy Alfaro, Sesme y Pavón, se recopilaran datos de las fichas clínicas de los pacientes en concordancia con el personal médico y de enfermería.

La población de estudio fue de 355 pacientes en la cual se utilizó técnica de muestra (sierra bravo) dando un resultado de muestra de 90 pacientes que fueron captados en 10 subcentros de salud de la zona norte de Manabi en Ecuador.

\section{Resultados}

Esta investigación se centra en la premisa que a pesar de las evidencias científicas de los efectos de la diabetes mellitus tipo 2, es necesario un adecuado nivel de conocimientos en estos pacientes sobre dicho padecimiento y su educación, para ello formaron parte del estudio 10 centros rurales de la provincia de Manabí. Este es un estudio pionero a nivel local, en el cual se valoraron estrategias y conocimientos de autocuidado en una población diabética tipo 2 .

Basados en los antecedentes nacionales e internacionales con la presente investigación se demostró un nivel bajo de conocimientos de la enfermedad en los pacientes, evidenciándose que el nivel de conocimiento de la población influye directamente en el cuidado que este tipo de paciente se dispensa así mismo y el aprovechamiento del mismo por parte del equipo de salud que los atiende directamente.

Entre los resultados más relevantes de esta investigación se resalta que un 5\% de la muestra respondió de forma positiva respecto a los si realizaban ajustes en sus hábitos de vida para mantenerse saludables; mientras que un $2 \%$ respondió negó hacerlo.

Un 5\% de la muestra estudiada da prioridad a las acciones que mantienen y protegen la salud; mientras que un $2 \%$ negó hacerlo. Un $4 \%$ de la muestra estudiada busca formas de cuidar su salud mientras

\begin{tabular}{|l|l|l|l|l|}
\hline PREGUNTAS & SI & \% & NO & \% \\
\hline $\begin{array}{l}\text { ¿Hago ajustes en mis hábitos de vida para mantenerme } \\
\text { Saludable? }\end{array}$ & & & & \\
& 77 & $5 \%$ & 13 & $2 \%$ \\
\hline
\end{tabular}

que un 5\% negó hacerlo. Un $4 \%$ respondió que se le alimentan de acuerdo a la su situación de salud y mantienen un peso adecuado; mientras que un $4 \%$ negó hacerlo

Un 7\% de la muestra no realiza ejercicios ni descansa durante el día mientras que un 3\% de la muestra si lo hace. Un 5\% no puede dormir lo suficiente para sentirse descansado mientras que un $4 \%$ si lo hace.

Un 5\% aseveró pedir información sobre su salud cuando no entiende lo que le recomiendan mientras que un 2\% no lo hace. Un 4\% de la población revisa su cuerpo para saber si hay algún cambio; mientras que un $4 \%$ no lo hace.

Un $4 \%$ de la población afirma haber sido capaz de cambiar hábitos no beneficiosos con tal de mejorar la salud; mientras que un 5\% no lo hace.

Un $4 \%$ de la población afirma pedir información sobre los nuevos medicamentes; mientras que un $4 \%$ no lo hace.

Un $4 \%$ de la población encuestada afirma buscar información si la salud se ve afectada.

La tabla 1 señala la aplicación de la escala de valoración sobre la capacidad de agencia de autocuidado de Ester Gallegos, ésta escala evalúa la capacidad de agencia que tiene el individuo para comprometerse con su autocuidado, en la cual la muestra captada señala que si realizan un sin número de actividades para el bienestar y autocuidado de la enfermedad que padecen. Los ajustes en los hábitos para mantenerse saludable, así como los cambios en el estilo de vida en cuanto a la alimentación, hábitos de higiene y actividad física o también el interés y necesidad de buscar información sobre su situación clínica son resultados que se pudieron identificar mediante esta escala. Otros aspectos como la capacidad del manejo de la enfermedad, las medidas de protección no solo individual sino familiar o el apoyo del entorno del paciente también se pudo valorar mediante esta escala. 


\begin{tabular}{|c|c|c|c|c|}
\hline $\begin{array}{l}\text { 2. Verifico si mis acciones y actitudes me protegen y } \\
\text { mantienen mi salud. }\end{array}$ & 61 & $4 \%$ & 29 & $5 \%$ \\
\hline $\begin{array}{l}\text { 3. ¿Hago cambios o ajustes de mi rutina si tengo problemas para realizar sus } \\
\text { haceres cotidiano }\end{array}$ & 59 & $4 \%$ & 31 & $6 \%$ \\
\hline 4. ¿Hago lo necesario para mantener limpio mi alrededor? & 75 & $5 \%$ & 15 & $3 \%$ \\
\hline $\begin{array}{l}\text { 5. Si es necesario, ¿doy prioridad a las acciones que mantienen y protegen mi } \\
\text { salud? }\end{array}$ & 77 & $5 \%$ & 13 & $2 \%$ \\
\hline 6. ¿Tengo la energía para cuidarme cómo debo? & 67 & $4 \%$ & 23 & $4 \%$ \\
\hline 7. ¿Busco mejores formas de cuidar mi salud? & 65 & $4 \%$ & 25 & $5 \%$ \\
\hline $\begin{array}{l}\text { 8. Con tal de mantenerme limpio, ¿realizo ajustes en cuanto a mi higiene } \\
\text { personal? }\end{array}$ & 75 & $5 \%$ & 15 & $3 \%$ \\
\hline $\begin{array}{l}\text { 9. ¿Me alimento de acuerdo a mi situación de salud y } \\
\text { mantengo un peso adecuado? }\end{array}$ & 70 & $4 \%$ & 20 & $4 \%$ \\
\hline $\begin{array}{l}\text { 10. ¿Tengo la capacidad de manejar las situaciones para } \\
\text { no perder el control y mantenerme en equilibrio? }\end{array}$ & 66 & $4 \%$ & 24 & $5 \%$ \\
\hline 11. ¿Hago ejercicio y descanso durante el día? & 52 & $3 \%$ & 38 & $7 \%$ \\
\hline $\begin{array}{l}\text { 12. ¿Tengo amigos a quienes puedo recurrir cuando } \\
\text { necesito ayuda? }\end{array}$ & 54 & $3 \%$ & 36 & $7 \%$ \\
\hline 13. ¿Puedo dormir lo suficiente para sentirme descansado? & 65 & $4 \%$ & 25 & $5 \%$ \\
\hline $\begin{array}{l}\text { 14. Cuando obtengo información sobre mi salud, ¿pido explicación sobre lo que } \\
\text { no entiendo? }\end{array}$ & 79 & $5 \%$ & 11 & $2 \%$ \\
\hline 15. ¿Reviso mi cuerpo para saber si hay algún cambio? & 67 & $4 \%$ & 23 & $4 \%$ \\
\hline $\begin{array}{l}\text { 16. He sido capaz de cambiar hábitos no beneficiosos con tal de mejorar mi } \\
\text { salud. }\end{array}$ & 64 & $4 \%$ & 26 & $5 \%$ \\
\hline $\begin{array}{l}\text { 17. Si tengo que tomar un nuevo medicamento ¿pido información sobre sus } \\
\text { efectos? }\end{array}$ & 67 & $4 \%$ & 23 & $4 \%$ \\
\hline $\begin{array}{l}\text { 18. ¿Tomo medidas para asegurar que mi familia y } \\
\text { yo estemos seguros? }\end{array}$ & 61 & $4 \%$ & 29 & $5 \%$ \\
\hline $\begin{array}{l}\text { 19. ¿Evalúo qué tan efectivo es lo que hago para } \\
\text { mantenerme con salud? }\end{array}$ & 73 & $4 \%$ & 17 & $3 \%$ \\
\hline 20. En mis actividades diarias, ¿me tomo un tiempo para cuidarme? & 61 & $4 \%$ & 29 & $5 \%$ \\
\hline $\begin{array}{l}\text { 21. ¿Busco información sobre mi salud si esta se ve } \\
\text { afectada? }\end{array}$ & 73 & $4 \%$ & 17 & $3 \%$ \\
\hline 22. ¿Busco ayuda si me encuentro con problemas de salud o de otro tipo? & 79 & $5 \%$ & 11 & $2 \%$ \\
\hline 23. ¿Dedico tiempo para mi mismo? & 66 & $4 \%$ & 24 & $5 \%$ \\
\hline $\begin{array}{l}\text { 24. Aun con problemas de salud soy } \\
\text { capaz de cuidarme }\end{array}$ & 74 & $5 \%$ & 16 & $3 \%$ \\
\hline
\end{tabular}

Tabla 1. Aplicación escala de valoración de la capacidad de agencia de autocuidado de Ester Gallegos (marque con una x la opción que lo identifique).

\section{Discusión}

La teoría de déficit de autocuidado de Dorotea Orem tiene tres subteorías relacionadas entre sí, cuyo objetivo principal es que el agente de autocuidado lleve a cabo y mantenga acciones para conservar la salud y la vida, se concuerda con los estudios realizados por los investigadores (Navarro \& Castro 2010). 
Dentro de la investigación se lograron detectar hábitos que contribuyen al autocuidado de los pacientes con Diabetes mellitus tipo 2, no obstante existen investigaciones que coinciden con los resultados expuestos y otras que muestran un lado diferente respecto al actuar de los pacientes que tal enfermedad.

De los 24 items que valoran al paciente diabético en el presente estudio, se observa en su totalidad una respuesta positiva por parte de la muestra; no obstante es necesario seguir abordando a este tipo de pacientes puesto que la clave del éxito de un buen autocuidado también se basa en la educación que el personal de salud puede brindarles, siendo esta oportuna y necesaria para poder seguir fomentando los hábitos y conductas correctos para el manejo de su enfermedad.

En el Distrito Federal de México se desarrolló una investigación similar donde exponen que con relación a las acciones de autocuidado que realizan los pacientes a favor del control de la enfermedad se encontró que, previo a la intervención, los pacientes desarrollaban $47.65 \%$ de las acciones y posterior a la capacitación se incrementaron a $57.61 \%$. A pesar de este incremento (10\%), el grupo continuó con un déficit importante de acciones, por lo que se concuerda con (Maya, 2008)

Otro hallazgo coincidente, es el de un estudio realizado en la parroquia Valle de Tucutunemo en Venezuela, donde más del $89 \%$ de los pacientes no presentaron autocuidado, pues excluyeron del tratamiento diabetológico la práctica de ejercicios físicos, ingestión de alimentos ricos en grasa y azúcares, no realizaron el lavado correcto de sus pies, ni ejecutaron con periodicidad sus análisis pertinentes, en la investigación se coincide con (Domínguez, 2004).

Según una investigación en la Universidad de Ciencias Médicas de Granma de la ciudad de Cuba, en donde se encuestaron 22 pacientes, llama la atención la ausencia de autocuidado en los pacientes diabéticos tipo 2 para un 90, $91 \%$. Las dificultades se ubican en relación con la práctica de ejercicio físico, la nutrición adecuada y el autocontrol de la glucemia, (Soler, 2016).

Autores como Sarrión Soro, expone que la educación diabetológica se debe priorizar en la intervención psicológica y que entre las temáticas a desarrollar se encuentran el afrontamiento a la enfermedad, la mejora de la adherencia al tratamiento y el control de la glucemia, aspectos que se relacionan con el autocuidado, se coincide con los estudios realizados por en este tema (Soro, 2010).

La educación participativa involucra al paciente como generador de su propio aprendizaje al establecer un vínculo entre la teoría y la práctica, con lo que se logra un efecto en la toma de decisiones sobre hábitos y estilos de vida saludables y, como consecuencia, en el control de la enfermedad, se concuerda con otras investigaciones (Fernández, 2012).

Otro estudio mexicano cuantificó la prevalencia del autocuidado de la salud en pacientes diabéticos tipo 2 y los factores de riesgo relacionados. La prevalencia de autocuidado encontrada fue del $62 \%$ el cual coincide con los reportes de la literatura. (Díaz, Márquez,\& Sabido 2007). Por ejemplo, en un estudio realizado en Estados Unidos, el $67 \%$ de los pacientes practicaba la revisión de los pies, el $60 \%$ realizaba ejercicio y el $46 \%$ prefería apegarse a la dieta. (Weinger, 2005)

Las prácticas de autocuidado relacionadas con la higiene personal y del entorno, el conocimiento acerca de la enfermedad y la adherencia a la dieta, son aspectos fundamentales para conservar una buena agencia de autocuidado. Se destaca además la importancia del apoyo social (familiar, programas grupales), recibido por el paciente con DM 2, como un factor para lograr adherencia al autocuidado (Herrera, 2012).

\section{Conclusiones}

El autocuidado de los pacientes con diabetes mellitus tipo 2 se ha visto reflejada en la presente investigación, dando a conocer que los hábitos y conductas actuales de la muestra son considerados como un indicador de un estilo de vida acorde para su patología, puesto que la realización de actividad física, los hábitos de higiene, la correcta alimentación, las horas de descanso y el interés del propio paciente en buscar ayuda o información acerca su patología evidencian las actividades del autocuidado en los pacientes con diabetes en beneficio a sí mismos.

\section{Bibliografía}

Díaz, A., Márquez, F. \& Sabido, A. (2007).Factores asociados al auto-cuidado de la salud en pacientes diabéticos tipo 2. Revista Archivos en Medicina Familiar, 9,(2) 99-107.

https://www.itsup.edu.ec/sinapsis 
Recuperado de https://www.redalyc.org/pdf/507/507114540 06.pdf

Domínguez, S. (2004). Intervención educativa en pacientes diabéticos en la parroquia Valle de Tucutunemo. Revista Médica Electrónica, 30,(2).Recuperado de http://www.revmatanzas.sld.cu/revista\%20m edica/ano\%202008/vol2\%202008/tema06.ht $\mathrm{m}$

Fernández, A. (2012). Estrategias de autocuidado en pacientes con diabetes mellitus tipo 2 Rev. Esp. Méd Quir, 17(2):94-99. Recuperado de https://www.medigraphic.com/pdfs/quirurgic as/rmq-2012/rmq122f.pdf

FPS. (2017). Mujeres y diabetes: Nuestro derecho a un futuro saludable. OPS-Federación de diabetes. Recuperado de https://www.paho.org/hq/index.php?option= com content $\&$ view=category \&layout=blog \&id=4475\&Itemid=40610\&lang=es

Hernández, R. , \& Aponte, L. (2014). Educación en diabetes: un aspecto clave de la formación actual en enfermería. Revista Orinoquía, 18 (2), 78-92. Recuperado de: https://dialnet.unirioja.es/servlet/articulo?cod igo $=5441155$

Herrera, A. (2012). Personas con diabetes mellitus tipo 2 y su capacidad de agencia de autocuidado, Cartagena. Revista Avances de Enfermería, 30, (2),39-46.Recuperado de https://revistas.unal.edu.co/index.php/avenfe $\underline{\mathrm{rm} / \mathrm{article} / \mathrm{view} / 36079 / 37474}$

Huañap, M. (2012). Maneras de enseñar el cuidarcuidado de enfermería en una universidad pública. Revista Ciencia y Tecnología, 8 , (22). Recuperado de http://revistas.unitru.edu.pe/index.php/PGM/ article/view/207/212

OPS. (2006). Ley Orgánica de Salud. Código Ética. Recuperado de https://www.paho.org/disasters/index.php?op tion=com_docman\&view=download\&catego ry_slug=safehospitalslegislation\&alias $=1770$ -ley-organica-de-salud2006\&Itemid=1179\&lang=en

Maya, A. (2008). Capacidades especializadas de autocuidado y adaptación de los pacientes con diabetes mellitus tipo 2. Revista CONAMED, 13.(2). Recuperado de https://dialnet.unirioja.es/servlet/articulo?cod igo $=3627021$

Ministerio de Salud Pública. Código de Ética. Ecuador. (2013). Recuperado de http://instituciones.msp.gob.ec/somossalud/i mages/documentos/guia/Doc_Codigo_Etica. pdf

Naranjo, Y. (2016). Diabetes mellitus: un reto para la salud pública. Revista Cubana de Enfermería, 32(1).Recuperado de http://scielo.sld.cu/pdf/enf/v32n1/enf01116.p df

Navarro, Y. \& Castro, M. (2010). Modelo de dorothea orem aplicado a un grupo comunitario a través del proceso de enfermería Global, 9, (2), 1-14.Recuperado de

https://www.redalyc.org/pdf/3658/36583475 5004.pdf

OMS. (2016). Informe mundial sobre la diabetes. Recuperado de https://www.who.int/diabetes/globalreport/es/

Soler, Y. (2016). Conocimientos y autocuidado en pacientes con diabetes mellitus tipo 2 . Revista Archivo Médico de Camagüey, 20, (3). Recuperado de http://revistaamc.sld.cu/index.php/amc/articl e/view/4295/2415

Soro, S. (2010). La SEEN explica los distintos tipos de diabetes a través de una campaña Medical Blogs. Recuperado de https://www.diariomedico.com/farmacia/dist ribucion/autocuidado/la-seen-explica-losdistintos-tipos-de-diabetes-traves-de-unacampana.html

Velásquez, M. (2014). Conocimiento y Practica del Autocuidado del profesional de Enfermería del Servicio Pediatra del Hospital Nacional Guillermo Almenara Irigoyen. Revista Cientifica de Ciencias de la Salud, 35-42.

Weinger, K. (2005). Measuring diabetes self-care: a psychometric analysis of the Self-Care Inventory-Revised. Diabetes Care., 1346-52. 
